\title{
Impact of hormonal therapy and other adjuvant therapies on contralateral breast volume change after implant-based breast reconstruction
}

\author{
Jung Youl Park, Jae-Ho Chung, Hyung Chul Lee, Byung-Il Lee, Seung-Ha Park, Eul-Sik Yoon \\ Department of Plastic and Reconstructive Surgery, Korea University College Medicine, Seoul, Korea
}

Background Adjuvant therapy after breast surgery, including tamoxifen or aromatase inhibitors, improves the postoperative outcomes and long-term survival of breast cancer patients. The aim of this study was to determine whether volume changes occurred in the contralateral breast during hormonal or other adjuvant therapies.

Methods This study reviewed 90 patients who underwent unilateral breast reconstruction between September 2012 and April 2018 using tissue expanders and a permanent implant after the surgical removal of breast cancer. The volume of the contralateral breast was measured using a cast before the first (tissue expander insertion) and second (permanent implant change) stages of surgery. Changes in breast volume were evaluated to determine whether adjuvant therapy such as hormonal therapy, chemotherapy, and radiation therapy influenced the volume of the contralateral breast.

Results The group receiving tamoxifen therapy demonstrated a significant decrease in volume compared with the group without tamoxifen $(-7.8 \%$ vs. $1.0 \% ; P=0.028)$. The aromatase inhibitor-treated group showed a significant increase in volume compared with those who did not receive therapy $(-6.2 \%$ vs. $4.5 \% ; P=0.023)$. There were no significant differences between groups treated with other hormonal therapy, chemotherapy, or radiation therapy.

Conclusions Patients who received tamoxifen therapy showed a significant decrease in volume in the contralateral breast, while no significant change in weight or body mass index was found. Our findings suggest that we should choose smaller implants for premenopausal patients, who have a high likelihood of receiving tamoxifen therapy.

Keywords Surgery, plastic / Reconstructive surgical procedures / Mammaplasty / Hormone antagonists / Tamoxifen

Received: 29 May 2018 • Revised: 29 Jul 2018 • Accepted: 27 Aug 2018

pISSN: 2234-6163 • elSSN: 2234-6171 • https://doi.org/10.5999/aps.2018.00563・ Arch Plast Surg 2018;45:432-440

\author{
Correspondence: Eul-Sik Yoon \\ Department of Plastic and \\ Reconstructive Surgery, Korea \\ University Anam Hospital, Korea \\ University College Medicine, 73 \\ Inchon-ro, Seongbuk-gu, Seoul \\ 02841 , Korea \\ Tel: +82-2-920-5368 \\ Fax: $+82-2-922-7437$ \\ E-mail: yesanam2@korea.ac.kr
}

\section{INTRODUCTION}

Surgical procedures are a mainstay of treatment for breast cancer, but adjuvant therapies improve the postoperative outcomes and long-term survival of breast cancer patients. Over $80 \%$ of patients overexpress estrogen receptors (ER) and/or progesterone receptors [1-4]. For these patients, adjuvant hormonal therapy should be used for at least 5 years, including selective estrogen receptor modulators (SERMs) such as tamoxifen or aromatase inhibitors (AIs) such as anastrozole and letrozole. It was 
also proven in a recent trial that for patients with ER-positive disease, continuation of tamoxifen therapy for 10 years, instead of 5 , reduced rates of recurrence and mortality [3].

Extensive mammographic density is strongly associated with the risk of breast cancer. SERMs such as tamoxifen are known to reduce the risk of breast cancer recurrence by affecting hormonal receptors and reducing mammographic density [5-8]. Cuzick et al. [6] and Nyante et al. [9] reported a 5\% to $10 \%$ decrease in mammographic density after 12 months of tamoxifen therapy.

Meanwhile, in patients who undergo breast reconstruction, hormonal therapy can cause breast volume to change, both by affecting the breast tissue itself and by affecting the fat distribution and body weight of the patient $[10,11]$. Ishii et al. [12] reported a significant decrease in breast volume in patients who received adjuvant therapy, especially in those with higher breast density. Theirs was the first study to report a breast volume decrease after adjuvant chemotherapy and tamoxifen therapy. However, a limitation of that study is that they used an uncommon method to measure breast volume [12].

The purpose of our study was to evaluate breast volume changes after hormonal therapy using a more reliable method than the previously mentioned study. Moreover, more patients were enrolled in this study to increase its reliability compared to that of the previous study [13]. Moreover, the relationships between hormonal therapy, body mass index (BMI), and breast volume were evaluated to explore effects on breast volume in more detail. The aim of this study was to observe whether volume changes in the contralateral breast occurred during hormonal therapy and other adjuvant therapies.

\section{METHODS}

A retrospective cohort study was performed with patients who underwent 2-stage breast reconstruction using tissue expanders, followed by placement of a permanent implant. Data were obtained from the Department of Plastic and Reconstructive Surgery of Korea University Anam Hospital between September 2012 and April 2018. Among the patients who underwent breast reconstruction surgery, 112 cases were reconstructed using tissue expanders followed by placement of a silicone implant. The following cases were excluded from the study: (1) bilateral reconstruction cases where both breasts were resected; (2) delayed reconstruction cases where adjuvant therapy began before the first operation; (3) secondary mastectomy cases due to local recurrence after breast-conserving therapy and/or additional contralateral breast cancer; and (4) non-elective cases in which properly collected data were not available. Ultimately, a total of 90 cases were included in our study.

Patient data were collected from the electronic medical records shared by oncologists and surgeons. The following data were collected: age, weight, BMI, hypertension, diabetes, smoking status, cancer type, hormone receptor status, hormonal therapy status, target therapy, preoperative and postoperative chemotherapy status, and radiation therapy.

Breast volume and photographic data were also collected for evaluation. Patients visited the office on the day prior to each operation for a breast volume measurement to be obtained. Breast volume was acquired using a cast by a single person (i.e., the breast coordinator) to reduce the error [13]. Photographic data were also acquired on the day before each operation. Informed consent was obtained from all patients for use of their photographic data. Institutional review board/ethics committee approval was obtained from the Institutional Review Board of Korea University Anam Hospital (K2018-1201-002).

When acquiring breast volume, the breast margin was first determined in the sitting position. By lifting up the breast, the upper margin could be identified. After drawing a line on the inframammary fold to establish the lower margin, a curvilinear line was drawn by connecting the upper and lower margins. Then, also with the patient in the sitting position, plastic wrap was applied and molded on the breast. Plastic tape was additionally applied to the wrap, to cast the molded wrap. The breast margin was drawn again on the applied cast before removing it from the breast. Finally, the cast was filled with water and breast volume was estimated by measuring the volume of the water that filled the cast (Fig. 1).

Immediate breast reconstruction was done using a tissue expander, at the same time when mastectomy was done by a breast surgeon. Approximately 6 months later, tissue expanders were changed to permanent silicone implants. The mean time interval between tissue expander insertion and implant change was 6.8 months (median, 5.8 months). Hormonal therapy was started, on average, 2.4 months after the mastectomy (median, 0.8 months). Hormonal therapy was applied for an average of 4.6 months, when patients were undergoing the second stage of surgery (median, 4.1 months).

The difference in the volume of the contralateral normal breast between the first stage and the second stage of reconstruction was measured. Differences in body weight and BMI between those periods were also assessed. These data were collected to evaluate any correlations among the type of adjuvant therapy, breast volume changes, and body weight changes. Proportional changes in breast volume and body weight were also calculated. The primary object of this study was to determine whether adjuvant therapy, including hormonal therapy, influenced breast 


\section{Fig. 1. Breast volume measured by plaster cast method}

(A) The breast margin was determined in the sitting position. By lifting up the breast, the upper margin could be identified. (B) Plastic wrap was applied on the breast and plastic tape was additionally applied on the wrap, to cast the molded wrap. (C) The breast margin was drawn again on the applied cast before removing it from the breast. (D) The cast was filled with water and breast volume was estimated by measuring the volume of the water that filled the cast.
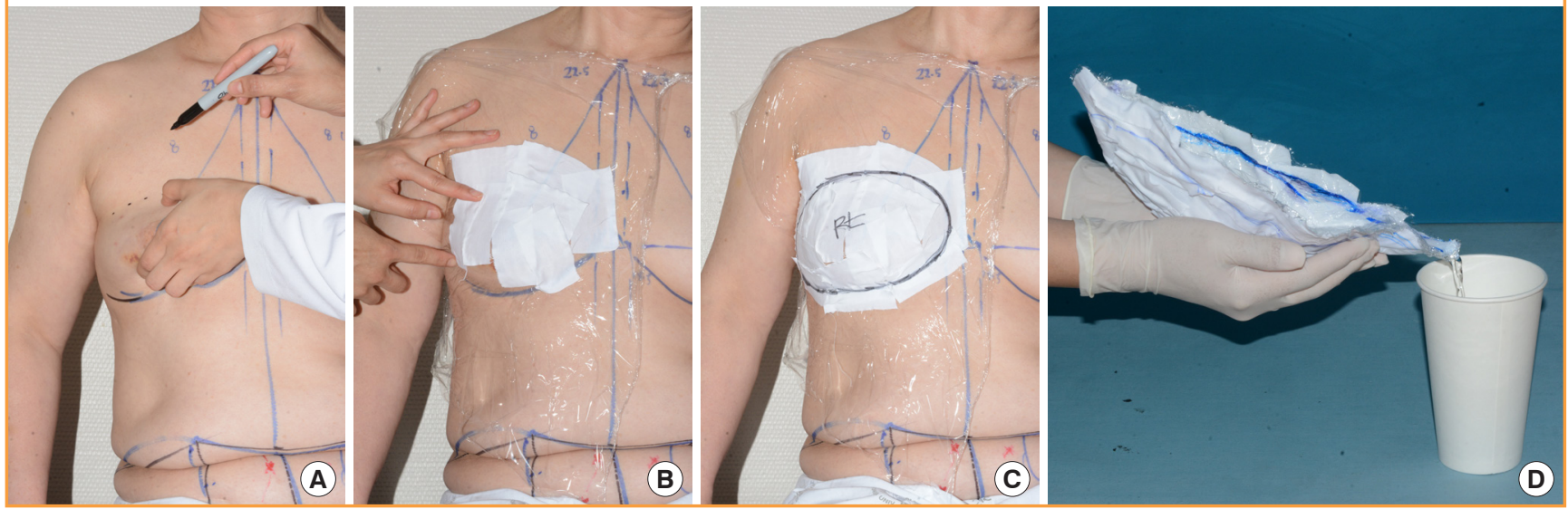

volume. We also aimed to assess whether adjuvant therapy had any impact on weight gain or loss, and to determine whether this change had an effect on breast volume.

\section{Statistical analysis}

Continuous variables are presented as means with the standard deviation, and as medians with interquartile ranges. The Kolmogorov-Smirnov test was used to check the normality of the data. The data we used for the analysis did not show a normal distribution according to the Kolmogorov-Smirnov test. All categorical data are presented as counts and percentages. For group comparisons, hypothesis testing was performed with the MannWhitney $U$ test for continuous data. Spearman correlation analysis was used to assess the correlation between breast volume and body weight changes. A 2-tailed value of $\mathrm{P}<0.05$ was used for all tests to indicate statistical significance. All analyses were performed using SPSS version 24 (IBM Corp., Armonk, NY, USA).

\section{RESULTS}

The mean age of the patients was $46.8 \pm 0.9$ years, and their mean BMI was $23.1 \pm 0.3 \mathrm{~kg} / \mathrm{m}^{2}$. Patient demographics and characteristics are presented in Table 1. Most patients (68\%) were diagnosed as having invasive ductal carcinoma, while $27 \%$ had ductal carcinoma in situ and $4 \%$ had invasive lobular carcinoma. Among the 77 patients (86\%) who had hormone receptor-positive tumors, 64 (71\%) received tamoxifen and 13 (14\%) received AIs. Additionally, goserelin (Zoladex; AstraZeneca, London, UK) and trastuzumab (Herceptin; Genentech, South San Francisco, USA) were administered to 18 patients (20\%)

\begin{tabular}{|c|c|}
\hline Characteristic & No. (\%) \\
\hline \multicolumn{2}{|l|}{ Patient demographics } \\
\hline No. of patients & 90 \\
\hline Age, mean $\pm S D$, yr & $46.8 \pm 0.9$ \\
\hline $\mathrm{BMl}$, mean $\pm \mathrm{SD}, \mathrm{kg} / \mathrm{m}^{2}$ & $23.1 \pm 0.3$ \\
\hline Hypertension & $10(11)$ \\
\hline Diabetes & $3(3)$ \\
\hline Smoking history & $8(9)$ \\
\hline \multicolumn{2}{|l|}{ Type of breast cancer } \\
\hline Ductal carcinoma in situ & $24(27)$ \\
\hline Invasive ductal carcinoma & $61(68)$ \\
\hline Invasive lobular carcinoma & $4(4)$ \\
\hline Phyllodes tumor & $1(1)$ \\
\hline \multicolumn{2}{|l|}{ HR status } \\
\hline HR-positive & $77(86)$ \\
\hline HER2-positive & $26(29)$ \\
\hline \multicolumn{2}{|l|}{ Hormone therapy } \\
\hline Tamoxifen & $64(71)$ \\
\hline Goserelin (Zoladex) & $18(20)$ \\
\hline Aromatase inhibitor & $13(14)$ \\
\hline \multicolumn{2}{|l|}{ Target therapy } \\
\hline Trastuzumab (Herceptin) & $18(20)$ \\
\hline Neoadjuvant chemotherapy & $10(11)$ \\
\hline Adjuvant chemotherapy & $36(40)$ \\
\hline Adjuvant radiation therapy & $10(11)$ \\
\hline
\end{tabular}

each. All 18 patients receiving goserelin were also treated with tamoxifen. Neoadjuvant chemotherapy was administered to $11 \%$ of the patients, and $40 \%$ of the patients underwent adjuvant chemotherapy. Radiation therapy was applied to $11 \%$ of patients.

The contralateral breast volume difference between the first and second operation was compared to the breast volume at the 
Table 2. Effect of adjuvant therapy on contralateral breast volume ratio $(\%)^{a)}$

\begin{tabular}{|c|c|c|c|c|c|c|c|}
\hline \multirow{2}{*}{ Adjuvant therapy } & \multicolumn{3}{|c|}{ No therapy } & \multicolumn{3}{|c|}{ Therapy } & \multirow{2}{*}{ P-value ${ }^{b)}$} \\
\hline & No. & Mean \pm SD & Median $(01,03)$ & No. & Mean \pm SD & Median $(01,03)$ & \\
\hline Tamoxifen & 26 & $1.0 \pm 15.8$ & $6.3(-9.3,13.4)$ & 64 & $-7.8 \pm 19.0$ & $-7.2(-19.1,6.5)$ & $0.028^{c)}$ \\
\hline Goserelin & 72 & $-5.0 \pm 18.8$ & $-3.3(-17.7,11.3)$ & 18 & $-6.2 \pm 17.9$ & $-3.1(-12.8,6.3)$ & 0.828 \\
\hline Aromatase inhibitor & 77 & $-6.9 \pm 18.7$ & $-5.8(-18.8,7.0)$ & 13 & $4.5 \pm 14.6$ & $7.4(0,14.3)$ & $0.023^{c)}$ \\
\hline Trastuzumab & 72 & $-6.1 \pm 17.9$ & $-4.0(-17.3,6.8)$ & 18 & $-1.7 \pm 21.1$ & $6.7(-17.8,12.1)$ & 0.316 \\
\hline Neoadjuvant CTx & 80 & $-5.8 \pm 19.1$ & $-3.8(-18.3,8.9)$ & 10 & $-1.1 \pm 13.2$ & $-2.9(-11.2,6.8)$ & 0.542 \\
\hline Adjuvant CTx & 54 & $-7.1 \pm 18.1$ & $-5.1(-17.1,6.3)$ & 36 & $-2.6 \pm 19.1$ & $3.7(-18.2,12.2)$ & 0.285 \\
\hline Adjuvant RTx & 80 & $-6.0 \pm 18.9$ & $-4.0(-18.8,7.4)$ & 10 & $1.7 \pm 14.4$ & $4.5(-13.2,12.2)$ & 0.256 \\
\hline Tamoxifen + Neo CTx & 85 & $-5.6 \pm 18.7$ & $-3.6(-17.5,7.7)$ & 5 & $0.1 \pm 17.1$ & $-2.9(-13.2,16.0)$ & 0.503 \\
\hline Tamoxifen + Adj CTx & 65 & $-5.4 \pm 18.2$ & $-3.6(-16.7,7.7)$ & 25 & $-4.9 \pm 19.9$ & $-2.9(-18.8,11.9)$ & 0.896 \\
\hline $\mathrm{Al}+\mathrm{Neo} \mathrm{CTX}$ & 89 & $-5.4 \pm 18.6$ & $-3.6(-17.5,8.3)$ & 1 & 6.4 & 6.4 & 0.551 \\
\hline $\mathrm{Al}+\mathrm{Adj} \mathrm{CTx}$ & 82 & $-6.5 \pm 18.7$ & $-4.6(-18.6,7.1)$ & 8 & $7.9 \pm 11.3$ & $10.6(4.8,14.7)$ & $0.022^{c)}$ \\
\hline
\end{tabular}

Table 3. Correlation between weight, BMI and contralateral breast volume change

\begin{tabular}{|lc|}
\hline Compared data & P-value $^{\text {a) }}$ \\
\hline Breast volume ratio vs. weight change ratio & 0.005 \\
Breast volume difference vs. BMI difference & 0.001 \\
\hline BMl, body mass index. \\
a)Spearman's correlation analysis was conducted for statistical analysis. \\
\hline
\end{tabular}

first operation. The ratio between the final breast volume and the initial breast volume was calculated. Patients receiving tamoxifen therapy demonstrated significantly decreased breast volume compared with patients who did not receive tamoxifen therapy $(-7.8 \%$ vs. $1.0 \% ; \mathrm{P}=0.028)$. The breast volume of the patients who received tamoxifen decreased by $25.4 \mathrm{~mL}$, whereas that of those who did not receive tamoxifen increased by $1.9 \mathrm{~mL}$ on average $(\mathrm{P}=0.019)$. On the contrary, patients treated with an AI showed a significant volume increase compared to those who did not receive an $\mathrm{AI}$ ( $4.5 \%$ vs. $-6.9 \% ; \mathrm{P}=0.023$ ). The use of an $\mathrm{AI}$ combined with adjuvant chemotherapy was also associated with a significant volume increase compared to those who did not receive that therapeutic combination $(7.9 \%$ vs. $-6.5 \%$; $\mathrm{P}=0.022)$. No statistically significant differences were found for the groups treated with other forms of hormonal therapy, such as goserelin $(\mathrm{P}=0.828)$ or trastuzumab $(\mathrm{P}=0.316)$. Chemotherapy and radiation therapy likewise showed no statistically significant effect on breast volume change (Table 2).

The correlation between weight change and breast volume change was evaluated, as weight change has been hypothesized to affect breast volume. The body weight of each patient at the time of the first and second operations was checked, and the difference was calculated. There was a significant correlation be- tween the proportional change in body weight and the breast volume change ratio $(\mathrm{P}=0.005)$, as well as between $\mathrm{BMI}$ and breast volume change $(\mathrm{P}=0.001)$ (Table 3$)$. The Spearman correlation coefficient of the proportional change in body weight and the breast volume change ratio was 0.293 , corresponding to a positive linear correlation. Patients who gained weight tended to experience an increase in breast volume (Fig. 2).

No statistically significant proportional change in body weight was found between patients who did or did not receive tamoxifen $(1.2 \%$ vs. $-0.3 \% ; P=0.135)$. Likewise, no other therapies examined in this study were associated with a significant difference in proportional body weight (Table 4).

Figs. 3 and 4 show preoperative photographs of the first and second stages of reconstruction surgery. Breast reconstruction was performed using a tissue expander, followed by a secondary permanent silicone implant. Total capsulectomy and a fat graft were performed during placement of the permanent implant. A contralateral balancing procedure using a silicone implant was performed to acquire additional symmetry.

\section{DISCUSSION}

This study shows that hormonal therapy affects breast volume. We first assumed that breast volume would decreased, either as a result of changes in breast density or due to weight loss, but believed that other minor factors could also affect volume. Compared to those who were not treated, tamoxifen-treated patients showed a significant decrease in breast volume, without significant changes in body weight. AIs, on the contrary, caused significant increases in breast volume without significantly impacting body weight. This demonstrates that breast volume 


\section{Fig. 2. Scatter plot of correlation}

Scatter plot of the correlation between proportional body weight change and the breast volume change ratio. The Spearman correlation coefficient was 0.293 , corresponding to a positive linear correlation.

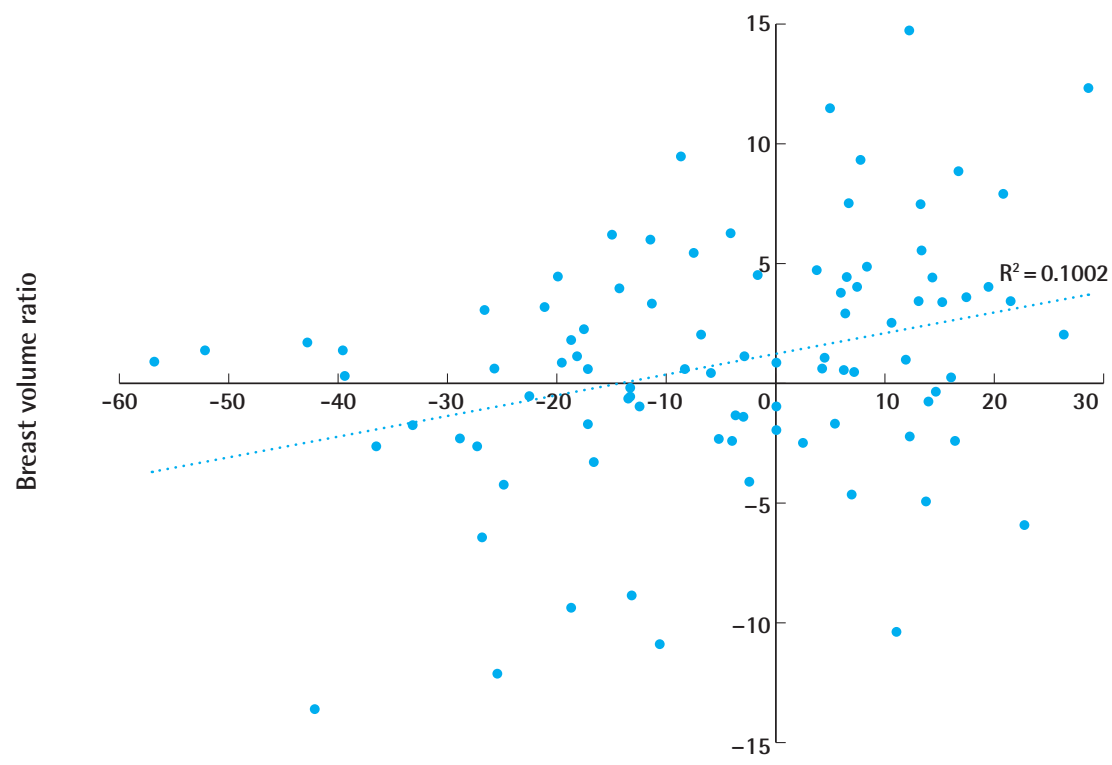

Proportional body weight change

\section{Table 4. Effect of adjuvant therapy on weight change (body mass index change) ratio (\%)}

\begin{tabular}{|c|c|c|c|c|c|c|c|}
\hline \multirow{2}{*}{ Adjuvant therapy } & \multicolumn{3}{|c|}{ No therapy } & \multicolumn{3}{|c|}{ Therapy } & \multirow{2}{*}{ P-value ${ }^{b)}$} \\
\hline & No. & Mean \pm SD & Median $(01,03)$ & No. & Mean \pm SD & Median $(01,03)$ & \\
\hline Tamoxifen & 26 & $-0.3 \pm 4.5$ & $-0.3(-2.4,3.2)$ & 64 & $1.2 \pm 5.2$ & $1.1(-1.1,3.9)$ & 0.135 \\
\hline Goserelin & 72 & $0.2 \pm 4.6$ & $0.6(-1.8,3.4)$ & 18 & $3.0 \pm 6.2$ & $2.8(-0.2,7.2)$ & 0.061 \\
\hline Aromatase inhibitor & 77 & $0.6 \pm 5.2$ & $0.6(-2.3,3.4)$ & 13 & $1.9 \pm 3.7$ & $2.8(-1.1,4.4)$ & 0.356 \\
\hline Trastuzumab & 72 & $0.4 \pm 4.7$ & $0.6(-1.8,3.3)$ & 18 & $2.4 \pm 6.0$ & $2.6(-1.7,5.1)$ & 0.155 \\
\hline Neoadjuvant CTx & 80 & $1.0 \pm 5.2$ & $0.8(-1.8,3.9)$ & 10 & $-0.7 \pm 3.8$ & $0.4(-2.2,1.6)$ & 0.304 \\
\hline Adjuvant CTx & 54 & $0.6 \pm 4.9$ & $0.5(-2.0,3.5)$ & 36 & $1.0 \pm 5.3$ & $1.0(-0.6,3.9)$ & 0.569 \\
\hline Adjuvant RTx & 80 & $0.6 \pm 4.9$ & $0.6(-2.0,3.4)$ & 10 & $2.5 \pm 6.2$ & $1.1(0.6,4.7)$ & 0.234 \\
\hline Tamoxifen + Neo CTx & 85 & $0.8 \pm 5.1$ & $0.7(-1.8,3.7)$ & 5 & $-0.4 \pm 5.0$ & $1.0(0.2,1.8)$ & 0.867 \\
\hline Tamoxifen + Adj CTx & 65 & $0.5 \pm 4.9$ & $0.5(-2.3,3.5)$ & 25 & $1.4 \pm 5.4$ & $1.1(0.2,3.9)$ & 0.375 \\
\hline $\mathrm{Al}+\mathrm{Neo} \mathrm{CTX}$ & 89 & $0.7 \pm 5.1$ & $0.7(-1.8,3.7)$ & 1 & 2.8 & 2.8 & 0.577 \\
\hline $\mathrm{Al}+\mathrm{Adj}$ CTx & 82 & $0.6 \pm 5.1$ & $0.6(-1.9,3.5)$ & 8 & $2.4 \pm 4.0$ & $3.1(0.5,4.5)$ & 0.239 \\
\hline \multicolumn{8}{|c|}{$\begin{array}{l}\text { SD, Standard deviation; Q1, first quartile; Q3, third quartile; CTx, Chemotherapy; RTx, radiation therapy; Neo CTx, neoadjuvant chemotherapy; Adj CTx, adjuvan } \\
\text { chemotherapy; Al, aromatase inhibitor. } \\
\text { a)Weight ratio = body mass index ratio = (patient's weight at implant change-patient's weight at expander insertion)/patient's weight at expander insertion; }{ }^{b} M a n n-W h i t n e y ~ \\
\text { test was conducted for statistical analysis. }\end{array}$} \\
\hline
\end{tabular}

changes after hormonal therapy resulted from changes in breast density itself, rather than changes in body weight.

Anti-estrogen agents block estrogen exposure by different mechanisms. Tamoxifen is a SERM, which acts as a competitive antagonist of the ER in breast tissue. Although tamoxifen acts as an antagonist in breast tissue, it selectively acts as an agonist in bone or endometrial tissue. SERMs are widely used for premenopausal women in whom ovarian estrogen production is still maintained $[1,14]$. Meanwhile, AIs are indicated for postmenopausal women in whom peripheral tissue conversion is the only source of estrogen. Aromatase is an enzyme found in subcutaneous fat, muscle, and breast tissue that transforms androgens into estrogens. AIs act by blocking the transformation in peripheral tissue of adrenal androgens into estrogens $[1,14]$. Goserelin, a gonadotropin-releasing hormone agonist that leads to ovarian suppression, is a less commonly used agent in pa- 


\section{Fig. 3. Case 1}

(A) Preoperative photograph of a 40-year-old female patient. The initial volume of the right breast was $350 \mathrm{~mL}$, and the patient's body weight was $54.2 \mathrm{~kg}$. (B) The right breast volume decreased to $250 \mathrm{~mL}$, whereas the patient's body weight was $55.1 \mathrm{~kg}$, after 6 months of tamoxifen therapy when permanent implant placement was performed. Additional total capsulectomy and a fat graft were performed on the right breast during the permanent implant placement procedure, after the photo was taken. A contralateral balancing procedure was not performed due to the patient's refusal.
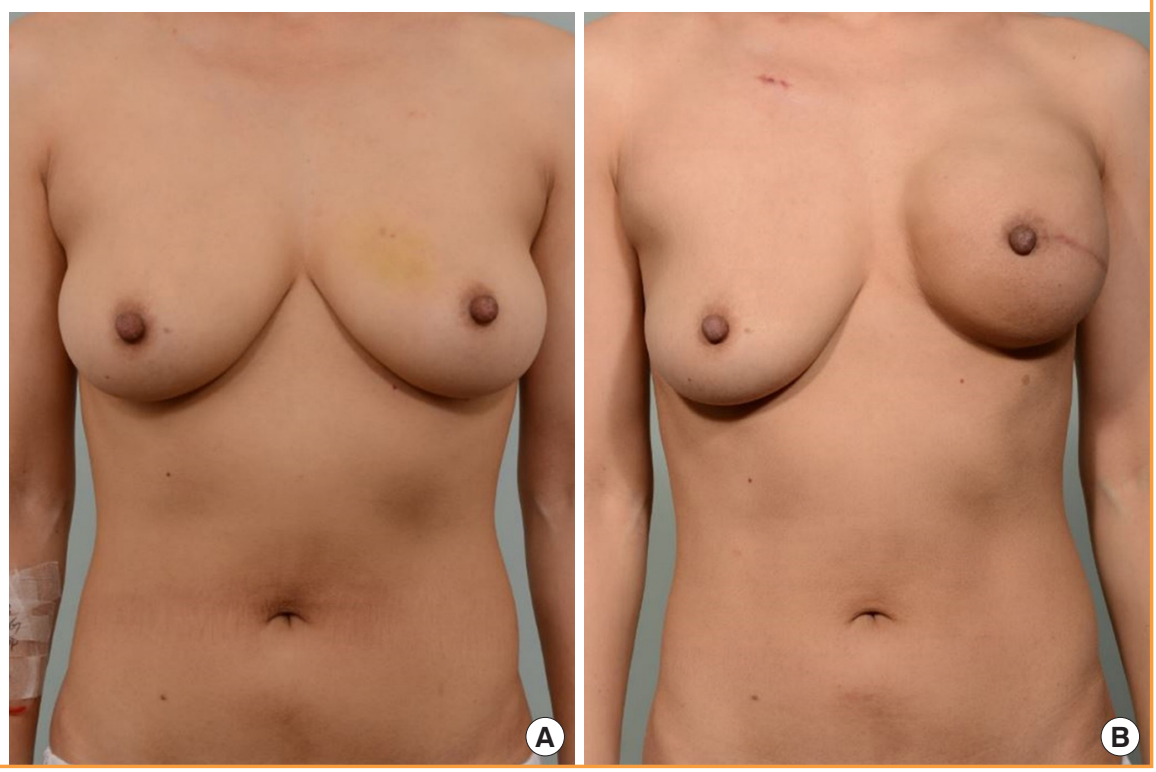

\section{Fig. 4. Case 2}

(A) Preoperative photograph of a 32-year-old female patient. The initial volume of the left breast was $455 \mathrm{~mL}$, and the patient's body weight was $60.6 \mathrm{~kg}$. (B) The left breast volume decreased to $395 \mathrm{~mL}$, with a body weight of $55.2 \mathrm{~kg}$, after 4 months of tamoxifen therapy when permanent implant placement was performed. Additional total capsulectomy and a fat graft were performed on the left breast during the permanent implant placement procedure, after the photo was taken. Additionally, a contralateral balancing procedure using a silicone implant was performed on the right breast at the same time. (C) A follow-up photo was taken after 9 months of tamoxifen therapy, when her weight was $53 \mathrm{~kg}$.
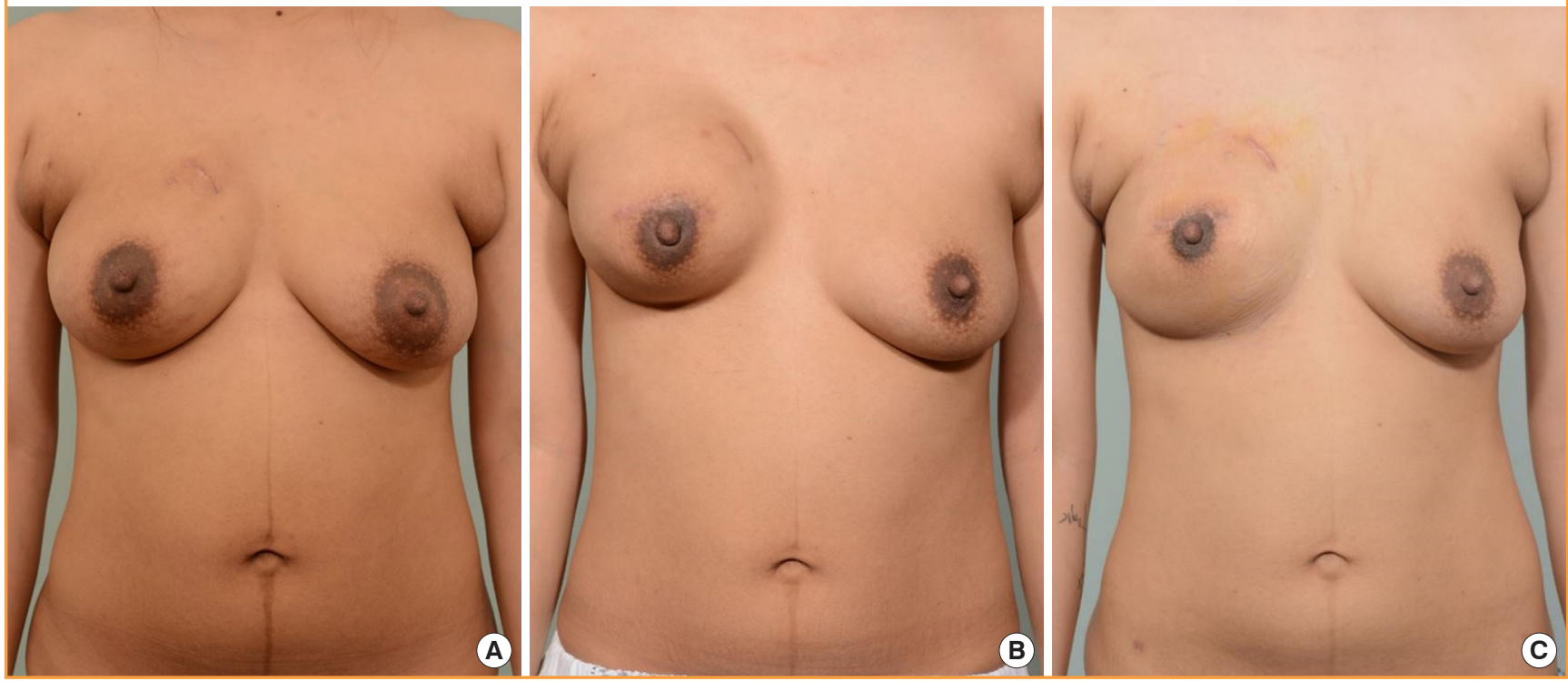

tients with breast cancer $[15,16]$. Nonetheless, goserelin therapy can be applied in premenopausal or perimenopausal women to prevent ovarian estrogen production [17].

In our study, tamoxifen was applied for an average of 4.8 months before patients underwent the second breast volume measurement. A $7.8 \%$ volume decrease was seen with a $1.2 \%$ increase in weight. According to our study, there was a linear correlation between breast volume increase and weight gain among the patients enrolled in the study. However, as there was no significant change in the weight of the patients treated with tamoxifen, factors other than weight influenced breast volume. Because tamoxifen is a selective ER antagonist in breast tissue, tamoxifen is expected to have more of an effect on breast tissue than on other peripheral tissues. By reducing mammographic density, tamoxifen treatment may be the major cause of changes in breast volume change $[5,8,18]$. 
After an average of 3.6 months of AI treatment, a $4.5 \%$ increase in breast volume and a $1.9 \%$ increase in weight were found. Previous studies from other groups have reported unclear findings regarding the relationship between $\mathrm{AI}$ treatment and breast density $[5,19]$. Since AIs are given to postmenopausal patients, however, breast volume may be related to weight gain, as it is known that menopause and estrogen deficiency are associated with weight gain $[11,20]$. Even though our study showed no significant weight gain to have resulted from AI therapy, there was a significant change in breast volume. It is difficult to explain the breast volume change after AI therapy, but fat distribution changes after menopause may be a possible explanation for this result. However, as far as we are aware, no study has reported additional fat deposition in breast tissue after menopause.

We also evaluated weight change in patients to exclude the effect of weight change on breast volume change. Although a positive correlation was found between weight change and breast volume change, there was no statically significant change in the proportional body weight of the patients after undergoing adjuvant therapy. The only noteworthy data for weight was found in patients who received goserelin, who experienced a near-significant amount of weight gain ( $3.0 \%$ vs. $0.2 \% ; \mathrm{P}=0.061)$. These results are relevant to other studies, as goserelin has been reported to lead to weight gain of $1 \%$ to $5 \%$ [11].

Although chemotherapy is associated with amenorrhea caused by ovarian dysfunction, Ishii et al. [12] reported no significant difference in breast volume between those who did or did not receive chemotherapy [21]. Likewise, in our study, we found no significant difference in breast volume according to whether the patient underwent chemotherapy. As radiation therapy is applied on the reconstructed breast, the effect on the contralateral breast is expected to be minimal. Our results also showed no significant difference in breast volume depending on whether the patient received radiation therapy.

Various methods for measuring breast volume have been introduced, but no single method is recognized as the best. Choppin et al. [22] reported that magnetic resonance imaging (MRI) and 3-dimensional (3D) scanning were the two most accurate methods for volume measurements, but the high cost of these devices and methods is a limitation. The Archimedes procedure and mammography have also been reported to be reliable, but mixed findings have been observed [23]. Although using a cast is less accurate than MRI or 3D scanning, it is still reliable considering its low cost [13]. The reliability for this measurement was enhanced in our study, as breast volume was measured by a single person. Ishii et al. [12] calculated breast volume by approximating the breast as a hemisphere (volume $=\pi \times$ width $\times$ height $x$ projection/6), but the reliability of this volume mea- suring method was not reported.

In unilateral breast reconstruction, balancing the breasts to obtain symmetry is an essential part of the procedure [24,25]. At the same time, this can be the most frustrating aspect of reconstruction for surgeons. Late revisions after single-stage breast reconstruction for asymmetry have been reported in $7.6 \%$ to $44 \%$ of patients [12]. Contralateral balancing augmentation or fat injection can be an option for asymmetric breasts; however, optimizing the aesthetic results in a single surgical procedure would be ideal.

Recently, more patients with breast cancer have received reconstructions using a direct-to-implant technique at our center. For these patients, the implant volume used for reconstruction should be determined with care, keeping in mind the shrinkage of the contralateral normal breast after tamoxifen therapy. Thus, we need to know whether the patient will receive tamoxifen therapy before the histologic diagnosis is reported. Previous studies have reported that $83 \%$ to $88 \%$ of all breast cancers are ER-positive [2-4]. Over $80 \%$ of premenopausal women would therefore receive tamoxifen therapy, because tamoxifen is the first-line adjuvant therapy for premenopausal women with ERpositive breast cancer. Thus, for unilateral breast reconstruction in premenopausal women, the majority of patients will experience a decrease in breast volume in the opposite breast after tamoxifen therapy. Our suggestion is to use smaller implants than the estimated size, especially when manufacturers do not have the precise implant size that is desired and surgeons need to choose between implants that are slightly larger or smaller than the intended size.

Previous reports have shown that breast density declines most in the first year after starting tamoxifen treatment [6]. Breast volume also decreases steeply in the first year. We cannot confirm the final volume decrease of the patients in the current study, but acquiring volume data from a longer follow-up period would provide additional information regarding volume changes. This would also give us more confidence regarding the size of the implant to be used in reconstructions in the future.

In our study, the patients who received tamoxifen therapy showed a significant volume decrease in the contralateral breast. No significant change in proportional body weight or BMI was found after tamoxifen therapy, which implies that weight change is not primarily responsible for the breast volume decrease after tamoxifen. Our study suggests that we should use smaller implants for premenopausal patients because over $80 \%$ of these patients are likely to receive tamoxifen therapy. However, longterm follow-up data of breast volume is needed to confirm the actual volume decrease after tamoxifen therapy. 


\section{NOTES}

\section{Conflict of interest}

No potential conflict of interest relevant to this article was reported.

\section{Ethical approval}

The study was approved by the Institutional Review Board of Korea University Anam Hospital (IRB No. K2018-1201-002) and performed in accordance with the principles of the Declaration of Helsinki. Written informed consents were obtained.

\section{Patient consent}

The patients provided written informed consent for the publication and the use of their images.

\section{REFERENCES}

1. Billon R, Bosc R, Belkacemi Y, et al. Impact of adjuvant antiestrogen therapies (tamoxifen and aromatase inhibitors) on perioperative outcomes of breast reconstruction. J Plast Reconstr Aesthet Surg 2017;70:1495-504.

2. Bouchard-Fortier A, Provencher L, Blanchette C, et al. Prognostic and predictive value of low estrogen receptor expression in breast cancer. Curr Oncol 2017;24:e106-14.

3. Davies C, Pan H, Godwin J, et al. Long-term effects of continuing adjuvant tamoxifen to 10 years versus stopping at 5 years after diagnosis of oestrogen receptor-positive breast cancer: ATLAS, a randomised trial. Lancet 2013;381:80516.

4. Ellis MJ, Coop A, Singh B, et al. Letrozole is more effective neoadjuvant endocrine therapy than tamoxifen for ErbB-1and/or ErbB-2-positive, estrogen receptor-positive primary breast cancer: evidence from a phase III randomized trial. J Clin Oncol 2001;19:3808-16.

5. Ekpo EU, Brennan PC, Mello-Thoms C, et al. Relationship between breast density and selective estrogen-receptor modulators, aromatase inhibitors, physical activity, and diet: a systematic review. Integr Cancer Ther 2016;15:127-44.

6. Cuzick J, Warwick J, Pinney E, et al. Tamoxifen-induced reduction in mammographic density and breast cancer risk reduction: a nested case-control study. J Natl Cancer Inst 2011; 103:744-52.

7. Boyd NF, Guo H, Martin LJ, et al. Mammographic density and the risk and detection of breast cancer. N Engl J Med 2007;356:227-36.

8. Schrading S, Schild H, Kuhr M, et al. Effects of tamoxifen and aromatase inhibitors on breast tissue enhancement in dynamic contrast-enhanced breast MR imaging: a longitudinal intraindividual cohort study. Radiology 2014;271:4555.

9. Nyante SJ, Sherman ME, Pfeiffer RM, et al. Longitudinal change in mammographic density among ER-positive breast cancer patients using tamoxifen. Cancer Epidemiol Biomarkers Prev 2016;25:212-6.

10. Feigelson HS, Jonas CR, Teras LR, et al. Weight gain, body mass index, hormone replacement therapy, and postmenopausal breast cancer in a large prospective study. Cancer Epidemiol Biomarkers Prev 2004;13:220-4.

11. Gambacciani M, Ciaponi M, Cappagli B, et al. Body weight, body fat distribution, and hormonal replacement therapy in early postmenopausal women. J Clin Endocrinol Metab 1997;82:414-7.

12. Ishii N, Ando J, Harao M, et al. Decreased contralateral breast volume after mastectomy, adjuvant chemotherapy, and anti-estrogen therapy, in particular in breasts with high density.J Plast Reconstr Aesthet Surg 2017;70:1363-8.

13. Bulstrode N, Bellamy E, Shrotria S. Breast volume assessment: comparing five different techniques. Breast 2001;10: 117-23.

14. Mirzabeigi MN, Nelson JA, Fischer JP, et al. Tamoxifen (selective estrogen-receptor modulators) and aromatase inhibitors as potential perioperative thrombotic risk factors in free flap breast reconstruction. Plast Reconstr Surg 2015;135: 670e-679e.

15. Jonat W, Kaufmann M, Sauerbrei W, et al. Goserelin versus cyclophosphamide, methotrexate, and fluorouracil as adjuvant therapy in premenopausal patients with node-positive breast cancer: the Zoladex Early Breast Cancer Research Association Study. J Clin Oncol 2002;20:4628-35.

16. Jakesz R, Hausmaninger H, Kubista E, et al. Randomized adjuvant trial of tamoxifen and goserelin versus cyclophosphamide, methotrexate, and fluorouracil: evidence for the superiority of treatment with endocrine blockade in premenopausal patients with hormone-responsive breast cancer: Austrian Breast and Colorectal Cancer Study Group Trial 5. J Clin Oncol 2002;20:4621-7.

17. Senkus E, Kyriakides S, Penault-Llorca F, et al. Primary breast cancer: ESMO clinical practice guidelines for diagnosis, treatment and follow-up. Ann Oncol 2013;24 Suppl 6: vi7-23.

18. Chen JH, Chang YC, Chang D, et al. Reduction of breast density following tamoxifen treatment evaluated by 3-D MRI: preliminary study. Magn Reson Imaging 2011;29:918.

19. Vachon CM, Suman VJ, Brandt KR, et al. Mammographic 
breast density response to aromatase inhibition. Clin Cancer Res 2013;19:2144-53.

20. den Tonkelaar I, Peeters PH, van Noord PA. Increase in breast size after menopause: prevalence and determinants. Maturitas 2004;48:51-7.

21. Zhou WB, Yin H, Liu XA, et al. Incidence of chemotherapyinduced amenorrhea associated with epirubicin, docetaxel and navelbine in younger breast cancer patients. BMC Cancer 2010;10:281.

22. Choppin SB, Wheat JS, Gee M, et al. The accuracy of breast volume measurement methods: a systematic review. Breast 2016;28:121-9.
23. Kayar R, Civelek S, Cobanoglu M, et al. Five methods of breast volume measurement: a comparative study of measurements of specimen volume in 30 mastectomy cases. Breast Cancer (Auckl) 2011;5:43-52.

24. Salgarello M, Visconti G, Barone-Adesi L, et al. Contralateral breast symmetrisation in immediate prosthetic breast reconstruction after unilateral nipple-sparing mastectomy: the tailored reduction/augmentation mammaplasty. Arch Plast Surg 2015;42:302-8.

25. Mioton LM, Jordan SW, Kim JY. A prospective analysis of dynamic loss of breast projection in tissue expander-implant reconstruction. Arch Plast Surg 2015;42:309-15. 